\title{
Wahlsiege ohne Stimmenmehrheit: Auswirkungen von verstärktem „Lagersplitting“" auf Mandatsverteilung und Koalitionsoptionen
}

\author{
Ossip Fürnberg und Danko Knothe
}

Das Wahlsystem der Bundesrepublik Deutschland wird überwiegend als vorbildlich dargestellt. Es gilt trotz seiner Komplexität als ausgewogener Kompromiss mit Modellcharakter, der das Beste der beiden Wahlrechtsformen Verhältniswahl und Mehrheitswahl vereine. ${ }^{1}$ Allerdings sind auch die Paradoxien des Wahlsystems wiederholt thematisiert worden. ${ }^{2}$ Tatsächlich kann das personalisierte Verhältniswahlrecht, anders als von Dieter Nohlen ${ }^{3}$ behauptet, ergebnisverzerrende und in hohem Umfang mandatsrelevante Effekte produzieren. Diese Folgen könnten sogar ein von Öffentlichkeit und Teilen der Wissenschaft wohl kaum für möglich gehaltenes Ausmaß erreichen. In diesem Beitrag soll mit Modellrechnungen darauf hingewiesen werden, dass Stimmensplitting innerhalb politischer Lager die Mehrheitsverhältnisse im Bundestag erheblich verschieben und unter Umständen letztlich sogar neue (von vielen splittenden Wählern möglicherweise nicht intendierte) Optionen zur Regierungsbildung schaffen kann. Mit der systematisch-experimentellen Untersuchung von potentiellen Auswirkungen, die durch verstärktes „Lagersplitting“ bei Bundestagswahlen entstehen könnten, werden Argumente für eine dringliche Reform des deutschen Wahlsystems unterstützt.

Aktuelle Bedeutung erfährt die Diskussion über den Verrechnungsmodus der Stimmen bei Bundestagswahlen durch das wegweisende Urteil des Bundesverfassungsgerichts (BVerfG) zum „negativen Stimmengewicht“. Die Verfassungsrichter haben angemahnt, dass das deutsche Wahlsystem „grundsätzlich frei von willkürlichen und widersinnigen Effekten sein “4 müsse. Maßgeblich sei der Grundsatz der Erfolgswertgleichheit aller Stimmen. Verzerrende Wirkungen mit direkter Mandatsrelevanz seien grundsätzlich fragwürdig und, sofern sie über einen gewissen Toleranzrahmen hinausreichend aufträten, inakzeptabel. Das BVerfG hat in seiner ständigen Spruchpraxis auch deutlich gemacht, dass eine zu große Anzahl von Überhangmandaten aufgrund der schwerwiegenden Verletzung des Grundsatzes der Erfolgswertgleichheit in jedem Fall als problematisch anzusehen ist.

Zwar sind die Gründe für die Entstehung von Überhangmandaten vielschichtig 5 , Stimmensplitting keineswegs die alleinige Ursache. Es kann aber festgehalten werden, dass es ohne die Möglichkeit, mit Erst- und Zweitstimme unterschiedliche Wahlakzente setzen zu

1 Vgl. stellvertretend Dieter Nohlen, Wahlrecht und Parteiensystem: zur Theorie und Empirie der Wahlsysteme, Opladen u.a. 2007.

2 Vgl. stellvertretend Martin Fehndrich, Paradoxien des Bundestags-Wahlsystems, in: Spektrum der Wissenschaft, 22. Jg. (1999), H. 2, S. 70 - 73, sowie zahlreiche Beiträge auf der Website www. wahlrecht.de.

3 „Überhangmandate führen (...) nicht zu einem grundsätzlichen Außerkraftsetzen des Proporzprinzips, indem sie etwa das Verhältnis zwischen Stimmen und Sitzanteil im Parlament grob verzerren." Dieter Nohlen, a.a.O. (Fn. 1), S. 329.

4 Vgl. Urteil des Zweiten Senats des BVerfG vom 3. Juli 2008, Az: 2 BvC 1/07, 2 BvC 7/07, Rn. 105.

5 Vgl. Joachim Behnke, The Strange Phenomenon of Surplus Seats in the German Electoral System, in: German Politics, 16. Jg. (2007), H. 4, S. 496 - 517. 
können, deutlich weniger Überhangmandate gäbe. Zudem tritt das Phänomen nur bei Bundestagswahlen auf: Die proporzverzerrenden Folgen sind hier vor allem deshalb problematisch, weil Überhangmandate nicht ausgeglichen werden. Gleichwohl kann ein starker Zusammenhang zwischen einem erhöhten Splittingniveau und Überhangmandaten vermutet werden.

Inzwischen splittet rund ein Viertel der Wähler bei Bundestagswahlen. Splitting ist also längst kein vernachlässigbares Randphänomen mehr. Für das hier vorzutragende Argument ist es völlig unerheblich, welche Präferenzen die Wähler verfolgen und aus welchen konkreten Gründen heraus sie sich für Splitting entscheiden - etwa wegen abnehmender Parteiidentifikation, aus Unkenntnis des Wahlsystems oder aufgrund einer expliziten Leihstimmenkampagne. ${ }^{6}$ Hier werden weder die Motive von Parteien, zum Splitting aufzurufen, noch die Splittingmotive der Wähler selbst untersucht. Im vorzustellenden Modell findet aus inhaltlichen und methodischen Gründen nur so genanntes Lagersplitting Berücksichtigung, das heißt die Verteilung von Erst- und Zweitstimmen auf Abgeordnete und Listenverbindungen von Parteien, die sich im Vorfeld der Wahl in einem politischen Lager verortet haben. Andere Splittingformen sind möglich und auch nicht unwahrscheinlich ${ }^{7}$, werden aber nicht behandelt. Explizite Koalitionsaussagen oder nicht dementierte Koalitionsabsichten der Parteien, wie sie für politische Lager angenommen werden können, erhöhen die Splittingwahrscheinlichkeit deutlich. Lagersplitting ist die am stärksten verbreitete Form geteilter Stimmabgabe. ${ }^{8}$ Aus dieser Feststellung resultiert unmittelbar die Frage, inwiefern sich die Mandatszahl eines Lagers durch stärkeres Splitting verändern kann. Ausgehend von der Tatsache, dass jede Form von Splitting mandatsrelevante Folgen hat, bietet sich die Untersuchung von Lagersplitting vor allem deshalb an, weil das Lager eine methodisch schlanke Möglichkeit bietet, Splittingeffekte experimentell zu isolieren und zu analysieren. Eine der bedeutsamsten Auswirkungen von verstärktem Lagersplitting könnte eine stärkere Stimmenungleichheit sein. In Extremfällen wären, weil sich die Fraktionsstärken im Bundestag massiv veränderten, Wahlerfolge ohne Stimmenmehrheit und neue Koalitionsoptionen möglich. Der an Wahlabenden oft angeführte „Wählerwille“ drohte damit ad absurdum geführt zu werden. Außerdem müsste bezweifelt werden, ob der vom Grundgesetz vorgeschriebene Verhältniswahlsystemcharakter bei einem hohen Anteil von Lagersplitting erhalten bliebe.

Unsere Darstellung ist um eine Art politikwissenschaftlicher „Technikfolgeabschätzung“ bemüht. Als Ausgangsmaterial liegt das Stimmenergebnis der Wahl zum 16. Deutschen Bundestag am 18. September 2005 zugrunde. Im Modell bleiben die Stimmenanteile der

6 Zur uferlosen Diskussion um die Motive vgl. unter anderem Harald Schoen, Stimmensplitting bei Bundestagswahlen: Eine Form taktischer Wahlentscheidung?, in: ZParl, 29. Jg. (1998), H. 2, S. 223 - 244; Paul W. Thurner, Taktisch oder aufrichtig? Zur Untersuchung des Stimmensplitting bei Bundestagswahlen, in: ZParl, 30. Jg. (1999), H. 1, S. 163 - 165 sowie Franz Urban Pappi / Paul W. Thurner, Electoral behavior in a two-vote-system: Incentives for ticket splitting in German Bundestag elections, in: European Journal of Political Research, 41. Jg. (2002), H. 2, S. 207 - 232.

7 Vgl. etwa Rüdiger Schmitt-Beck, Denn sie wissen nicht, was sie tun ... Zum Verständnis des Verfahrens der Bundestagswahl bei westdeutschen und ostdeutschen Wählern, in: ZParl, 24. Jg. (1993), H. 2, S. $393-415$.

8 Vgl. Franz Urban Pappi / Alexander Herzog / RalfSchmidt, Koalitionssignale und die Kombination von Erst- und Zweitstimme bei den Bundestagswahlen 1953 bis 2005, in: ZParl, 37. Jg. (2006), H. 3, S. $493-513$. 
Lager Rot-Grün (SPD, Bündnis 90/Grüne) und Schwarz-Gelb (CDU/CSU, FDP) und auch die der Linkspartei unverändert. Mit anderen Worten: kein Lager verfügt im Modell in der Summe über mehr Stimmen, als für seine Parteien tatsächlich abgegeben worden sind. Manipuliert wurde lediglich die Verteilung der Stimmen innerhalb der beiden politischen Lager. Die Analysekategorie „politisches Lager“ erscheint aus zwei Gründen angebracht: Erstens spiegeln sich darin offenkundige, über Jahre hinweg stabile parteipolitische Präferenzen der Wähler, und zweitens haben sich die Parteien durch Koalitionsaussagen und wahlkampfpolitische Akzente wiederholt in Lagern, oder anders ausgedrückt in „preelectoral coalitions" ${ }^{\text {"9 }}$, verortet. Dies galt auch bei der Bundestagswahl 2005, in der sich die rot-grüne Regierungsmehrheit der Herausforderung durch die schwarz-gelbe Opposition zu stellen hatte. Sowohl SPD und Bündnis90/Die Grünen als auch Union und FDP machten im Wahlkampf eindeutige Koalitionsaussagen. Anhänger dieser Parteien gaben einer Zweierkoalition „ihres“ Lagers deutlich den Vorzug gegenüber anderen Koalitionsoptionen, und viele Wähler haben die Selbstverortung der Parteien in ihrem Wahlverhalten nachvollzogen. Laut repräsentativer Wahlstatistik zeigt das Wahlverhalten eine deutliche Lagertendenz. Über sieben Prozent aller Wähler splitteten zwischen CDU/CSU und FDP, für ein Splitting im rot-grünen Lager entschieden sich über fünf Prozent. Damit splitteten mehr als die Hälfte der Wähler, die von der Möglichkeit geteilter Stimmabgabe Gebrauch machten, innerhalb der politischen Lager. Bemerkenswert ist außerdem, dass der Großteil (über 85 Prozent) dieser Wähler strategisch rational handelte, also die Erststimme der großen, die Zweitstimme der kleinen Partei gab. ${ }^{10}$

\section{Geteilte Stimmabgabe und Überhangmandate: ein wahlsystematischer Webfehler}

Nach Artikel 38 GG sollen die Abgeordneten des Bundestages nach den Grundsätzen einer allgemeinen, unmittelbaren, freien, gleichen und geheimen Wahl gewählt werden. Das genaue Verfahren wird durch das Bundeswahlgesetz geregelt. Bei Bundestagswahlen hat jeder Wähler zwei Stimmen, die er jeweils für einen Direktkandidaten im Wahlkreis und eine Landesliste abgeben kann $(\$ 4$ BWG). Die Direktkandidaten in den Wahlkreisen werden nach dem Verfahren der relativen Mehrheitswahl bestimmt. Für die Verrechnung der Zweitstimmen wird nach Proporz mittels Divisorverfahren ermittelt, wie viele Sitze insgesamt auf die Landeslisten der Parteien entfallen (Oberverteilung). Die Verteilung der Mandate auf die Landeslisten erfolgt anschließend (Unterverteilung) nach dem gleichen Verfahren, nach dem bereits die Mandate auf die einzelnen Parteilisten verteilt wurden. Die in einem Land errungenen Direktmandate werden von den einer Liste zustehenden Mandaten abgezogen. Die restlichen Mandate werden durch die Listen besetzt. Wenn eine Partei in einem Bundesland mehr Direktmandate errungen hat, als ihrer Liste nach Proporz zugeteilt wurden, gelten die überschüssigen Mandate als Überhangmandate und bleiben der Partei erhalten. Die Anzahl der Sitze im Bundestag erhöht sich um die Anzahl der entstandenen Überhangmandate.

In $₫ 6$ Absatz 1 BWG finden sich Hinweise auf Situationen, in denen Wähler nicht mit der Erst- und Zweitstimme die selbe Parteipräferenz bekunden. Explizit werden nur die

9 Vgl. Sona N. Golder, The Logic of Pre-electoral Coalition Formation, Columbus 2006.

10 Vgl. Franz Urban Pappi / Alexander Herzog / Ralf Schmidt, a.a.O. (Fn. 8), S. 502 ff. 
Fälle erwähnt, bei denen ein Wähler einen „unabhängigen“ Direktkandidaten oder den Kandidaten einer Partei wählt, die keine Landesliste in dem betreffenden Bundesland aufgestellt hat. Ist ein Wähler in einem dieser Fälle mit seiner Erststimme erfolgreich, das heißt, zieht der Direktkandidat in den Bundestag ein, kann seine Zweitstimme nicht in die Verrechnung der Zweitstimmen eingehen, die über die Mandatsverteilung nach Proporz im Bundestag entscheidet. Die Zweitstimme verfällt also. Darüber hinaus werden die über Proporz zu vergebenden Mandate um die Anzahl der auf diesem Weg erfolgreichen Wahlkreiskandidaten verringert. Dieser Fall trat erst einmal auf. Bei der Bundestagswahl 2002 wurden zwei Berliner PDS-Kandidaten direkt in den Bundestag gewählt und die Gesamtzahl der bei der Proporzberechnung zugrunde gelegten Mandate entsprechend verringert (auf 596). Nach wahlsystematischer Logik hätten aber auch die berühmt gewordenen „Berliner Zweitstimmen " nicht bei der Proporzberechnung berücksichtigt werden dürfen. ${ }^{11}$

Stimmensplitting wird im BWG so behandelt, dass die Nichtberücksichtigung von Zweitstimmen bereits erfolgreicher Direktkandidaten zwingend erforderlich ist - sie erfolgt analog zur Verrechnung von Direktmandaten mit zustehenden Listenmandaten. Offenbar wollte der Gesetzgeber ausdrücklich verhindern, dass ein Wähler mit beiden Stimmen erfolgreich auf die Mandatsverteilung im Bundestag einwirken kann. Die inzwischen als Normalfall zu betrachtende Form von Stimmensplitting, bei der ein Wähler seine Stimme auf zwei mit Landeslisten antretenden Parteien aufteilt, wird bemerkenswerterweise im BWG nicht einmal erwähnt. Dass Erst- und Zweitstimmen von Wählern, die ihre Stimmen Parteikandidaten und Parteilisten unterschiedlicher Couleur geben, bei der Proporzverteilung der Mandate nicht miteinander verrechnet werden, macht deutlich, dass Parteiensplitting im Gegensatz zur Wahl unabhängiger Wahlkreiskandidaten entweder nicht vorgesehen ist oder als irrelevant erachtet wurde. Wahlsystematisch ist es jedoch nicht unproblematisch, dass die Wähler mit Erst- und Zweitstimme unterschiedliche Präferenzen bekunden können. Auf die möglicherweise vorliegende Verfassungswidrigkeit ist wiederholt hingewiesen worden. ${ }^{12}$ Nur Parteiensplitting eröffnet die Möglichkeit, mit beiden Stimmen erfolgreich Einfluss zu nehmen, was verfassungsrechtlich fragwürdig erscheint. ${ }^{13}$ Die bedeutsamste

11 Das Bundesverfassungsgericht hatte aufgrund einer Wahlprüfungsbeschwerde 2005 eine nochmalige Auszählung der Stimmen veranlasst, die Beschwerde letztlich aber mit der salomonischen Entscheidung verworfen, dass keine Mandatserheblichkeit festzustellen gewesen sei. Unabhängig davon erscheint die vom Bundeswahlleiter vertretene Rechtsauffassung bezüglich der Zweitstimmenberücksichtigung diskussionswürdig. Bei einer Nichtberücksichtigung hätten sich, wie das Ergebnis der Nachauszählung ergab, tatsächlich keine Mandatsverschiebungen ergeben. Allerdings hätte die SPD aufgrund von Überhangmandaten erstmals die größte Fraktion gestellt, ohne die meisten Zweitstimmen errungen zu haben (der Rückstand auf die Union hätte 20360 Stimmen betragen).

12 Vgl. unter anderem Markus Winkler, Gleichheit der Wahl, „Stimmensplitting“ und Überhangmandate, in: Recht und Politik, 35. Jg. (1999), H. 4, S. 244 - 247; Wolfgang Schreiber, Handbuch des Wahlrechts zum Deutschen Bundestag, Köln u.a. 2002, S. 215, siehe auch Helmut Niclaus, Die unzulängliche Rechtfertigung der Überhangmandate: Aufklärungsversuche, in: ZParl, 27. Jg. (1996), H. 3, S. 383 - 393.

13 Nur Parteiensplitting eröffnet die Möglichkeit mit beiden Stimmen erfolgreich Einfluss zu nehmen, weil die jeweiligen Zweitstimmen nicht wie bei einer einheitlichen Stimmenabgabe mit den erfolgreichen Erststimmen verrechnet oder wie bei der erfolgreichen Wahl unabhängiger Direktkandidaten gestrichen werden. Diese Bevorzugung des Parteiensplittings erscheint verfassungsrechtlich fragwürdig, vgl. Markus Winkler, a.a.O. (Fn. 12), S. 245. 
Folge von Parteiensplitting können Überhangmandate sein, die auf der Landesebene entstehen und im deutschen Wahlsystem auf Bundesebene nicht ausgeglichen werden. Hinzu kommen im nur von wenigen Experten verstandenen Verrechnungsverfahren andere Effekte wie das negative Stimmengewicht, die ebenfalls verfassungsrechtliche Zweifel geweckt haben. ${ }^{14}$ Die Möglichkeit der doppelten Stimmabgabe scheint nicht nur viele Wähler zu verwirren $^{15}$, sondern entfaltet eine Vielzahl verfassungsrechtlich bedenklicher Implikationen. Ohne die Splittingmöglichkeit wären nahezu alle in den zahlreichen wahlrechtsbezogenen Bundesverfassungsgerichtsverfahren geäußerten Zweifel an der Verfassungsmäßigkeit des deutschen Wahlrechts praktisch gegenstandslos.

Parteiensplitting bildet ein Einfallstor für Einflussmaximierung (der splittenden Wähler) und Proporzverzerrung (im Bundestag). Im Extremfall könnten 299 Überhangmandate vergeben werden und darüber hinaus noch einmal 598 Mandate nach dem Verrechnungsverfahren der Zweitstimmen. Dieser Fall träte ein, wenn bei allen auf Wahlkreisebene erfolgreichen Direktkandidaten auf die zugehörigen Listenverbindungen im jeweiligen Bundesland keine oder nur sehr wenige Zweitstimmen entfielen. ${ }^{16}$ Wahlsystematisch können also bei der Bundestagswahl nicht nur 598 Mandatsträger von den Wählern bestimmt werden, sondern maximal 897. Es sind genau diese „Schattenmandate“, die den Effekt kippender Mehrheiten bewirken können. ${ }^{17}$ Abhängig davon, wie stark dieser Pool ausgeschöpft wird und welche Parteien dabei besonders profitieren, können sich Mehrheitsverhältnisse im Bundestag verändern und unter Umständen neue Regierungsoptionen ergeben. Dass die Schattenmandate in der Praxis niemals vollständig ausgeschöpft werden dürften, kann kein Argument dafür sei, die Problematik zu ignorieren. Eine Ausnutzung taktischen Stimmensplittings zur Erzeugung von Überhangmandaten ist zumindest im weiter unten diskutierten Fall der Nachwahl in Dresden 2005 empirisch bereits nachweisbar.

\section{Modell und Datenbasis}

Empirische Basis des Modells ist das Ergebnis der Bundestagswahl 2005. ${ }^{18}$ Die auf FDP und Unionsparteien entfallenen Stimmen werden auf Ebene der 299 Wahlkreise summiert. Die jeweils zusammengefassten Erst- und Zweitstimmen werden als Erst- und Zweitstimmen des schwarz-gelben Lagers modelliert. Dabei werden die auf CSU und CDU entfallenden Mandate unter dem Begriff „Union“ angegeben, beide Parteien aber bei der Stimmenverrechnung (wie nach BWG notwendig) getrennt behandelt. Analog werden die Stimmen von SPD und Bündis90/Die Grünen zu Erst- und Zweitstimmen des rot-grünen

14 Vgl. stellvertretend das bereits erwähnte Urteil des Bundeverfassungsgerichts zum negativen Stimmgewicht (Fn. 4).

15 Vgl. Rüdiger Schmitt-Beck, a.a.O. (Fn. 7).

16 Dieser Hinweis ist wichtig: Theoretisch könnte es - durch regional stark unterschiedliche Wahlergebnisse - dazu kommen, dass viele unterschiedliche Parteien Überhangmandate erzielen, und zusätzlich durch Zweitstimmen außerhalb ihrer Hochburgen (also in anderen Bundesländern) an der Proporzverteilung teilnehmen könnten.

17 Diesen Aspekt von Überhangmandaten thematisiert auch Joachim Behnke, a.a.O. (Fn. 5), S. 497. Dort finden sich auch weiterführende Literaturverweise.

18 Die abgegebenen Erst- und Zweitstimmen sind, auf die Ebene der Wahlkreise heruntergebrochen, auf der Homepage des Bundeswahlleiters frei verfügbar, vgl. www.bundeswahlleiter.de/bundestagswahl2005/ergebnisse/bundesergebnisse (Abruf am 1. Februar 2009). 
Lagers zusammengefasst. Wichtig ist der Hinweis, dass die Gesamtstimmenanteile der politischen Lager (und die der Linkspartei) für sich konstant gehalten werden. Kein Lager erhält in unserem Modell mehr Stimmen als im tatsächlichen Wahlergebnis ausgewiesen.

Anhand des empirischen Materials sollen experimentell erhöhte Splittingniveaus in politischen Lagern systematisch untersucht werden. Entsprechend der Fragestellung interessieren nicht individuelle Verhaltensweisen, sondern die Effekte auf der Aggregatebene. Dabei stehen allein die Auswirkungen von „strategisch rationalem “19 Lagersplitting im Fokus, bei dem Wähler ihre Erststimme dem aussichtsreichen Direktkandidaten einer großen Volkspartei und die Zweitstimme einer politisch nahestehenden kleinen Partei geben. Damit lassen sich mandatsrelevante Auswirkungen experimentell sehr gut isolieren: Bei unveränderter Gesamtstimmenanzahl des Lagers dürften alle auftretenden Veränderungen zum tatsächlichen Ergebnis 2005, also eine veränderte Anzahl der auf das Lager entfallenden Mandate, allein dem höheren Splittingniveau zuzuschreiben sein. Im Modell wird Splittingverhalten über die Lagergrenzen hinweg ausgeblendet, auch wenn es in der Realität selbstverständlich vorkommt und die dargestellten Effekte wahrscheinlich abmildern dürfte. Insofern sind die auf geteilte Stimmabgabe zurückzuführenden Auswirkungen auf Mandatsverteilung und Mehrheitsbildung im Modell deutlich überzeichnet.

Zur Modellierung steigenden Splittings ist es notwendig, zunächst das tatsächliche Ausgangsniveau zu bestimmen. Bei der Bundestagswahl 2005 wurde der Splittinganteil auf etwa 25 Prozent geschätzt. Trotz dieser Schätzungen mittels repräsentativer Wahlstatistik und durch Umfrageinstitute ist schwer zu ermitteln, in welchem Umfang welche Splittingkombination in den Wahlkreisen jeweils gewählt wurde. ${ }^{20}$ Dieser Sachverhalt macht es nahezu unmöglich, Wahlergebnisse zu modellieren, in denen das Stimmensplitting von einem Ausgangsniveau von geschätzten 25 Prozent steigt. Aus diesem Grund sollen hier zwei Extrempunkte festgelegt werden, zwischen denen Stimmensplitting stärker als im Wahlergebnis bereits abgebildet stattfinden kann: Der Nullpunkt ist das vom Bundeswahlleiter ausgewiesene Wahlergebnis. Von diesem Punkt ausgehend werden im Modell die Erst- und Zweitstimmen eines Lagers auf Wahlkreisebene umverteilt. Dies geschieht in mehreren Schritten so lange, bis die (zunächst) große Partei eines politischen Lagers alle Erst- und die (zunächst) kleinere Partei alle Zweitstimmen erhalten haben. Obwohl es plausibel wäre, dass Anhänger der kleinen Lagerpartner tendenziell eher splitten und ihre Erststimme dem großen Lagerpartner geben ${ }^{21}$, wissen wir nicht genau, wie sich das Verhältnis der Erst- und Zweitstimmenübertragung im Lager bei ansteigendem Splittingniveau entwickeln würde. Deswegen wird in jedem Wahlkreis der gleiche Prozentanteil von Erststimmen der kleinen Partei auf die große wie von Zweitstimmen der großen auf die kleine Partei übertragen.

19 Der Begriff „strategisch rational“ steht synonym für „strategisches“ Wählen und bezieht sich ausdrücklich nur auf den Akt der Stimmabgabe selbst; ähnlich Thomas Gschwend, Ticket-Splitting and Strategic Voting under mixed Electoral Rules: Evidence from Germany, in: European Journal of Political Research, 46. Jg. (2007), H. 1, S. 1 - 24 und Franz Urban Pappi / Alexander Herzog / Ralf Schmidt, a.a.O. (Fn. 8).

20 Auch eine ökologische Regression kann hier nur annäherungsweise weiterhelfen. Vgl. dazu die Ergebnisse von Thomas Gschwend, Strategic Voting in Mixed Electoral Systems, Wiesbaden 2004.

21 So argumentieren zum Beispiel Joachim Behnke / Stefanie Hergert / Florian Bader, Stimmensplitting - Kalkuliertes Wahlverhalten unter den Bedingungen der Ignoranz, Bamberger Beiträge zur Politikwissenschaft, Nr. I-7, Bamberg 2004, S. 55. 
Wohlgemerkt handelt es sich dabei um zusätzliche Splittingeffekte, die über die im Wahlergebnis bereits vorhandenen hinausgehen. Sprechen wir also von einer Zunahme des Splittingniveaus im schwarz-gelben Lager um zehn Prozent, wird in allen 299 Wahlkreisen eine Übertragung von zehn Prozent der Erststimmen von der FDP auf die Unionsparteien modelliert, gleichzeitig ein Transfer von zehn Prozent der Zweitstimmen von den Unionsparteien zur FDP.

Selbstverständlich wählt nur ein geringer Teil der Wähler nach diesen Modellannahmen. Gleichwohl hat strategisches Lagersplitting bei den Bundestagswahlen der jüngeren Vergangenheit eine immer größere Rolle gespielt. ${ }^{22}$ Die Modellierung vernachlässigt bewusst, dass die auf ein Lager entfallenden Erst- und Zweitstimmen, die im Modell transferiert werden, natürlich nicht nur von Lageranhängern abgegeben werden. Ausweislich der repräsentativen Wahlstatistik hat 2005 jedoch nur eine Minderheit unter den Splittern ihre Stimmen nicht lagerkonform verteilt. Die Nichtberücksichtigung dieses Phänomens ist dem Tradeoff zwischen Realitätsnähe und einem möglichst einfachen Modell geschuldet. Um das Modell schlank zu halten, haben wir außerdem darauf verzichtet, regionale Unterschiede im Parteiensystem (Hochburgeneffekte, effektive Parteienzahl) und unterschiedliche Niveaus bei der Wahlbeteiligung zu berücksichtigen, obwohl beide Faktoren die Entstehung von Überhangmandaten stark beeinflussen können. Bekannt ist beispielsweise, dass in Bundesländern, wo Überhangmandate besonders häufig auftreten, auch häufiger als im Bundesschnitt gesplittet wird. Lerneffekte im Elektorat und eine Splittingzunahme aufgrund von Erfahrungswerten sind also begründet zu vermuten, dennoch werden im Modell alle Wahlkreise gleich behandelt.

Die Konzentration auf den Lagereffekt erklärt sich in erster Linie damit, dass die Einflüsse von verstärkter „Vorbereitung“ des Elektorats durch Signale der politischen Parteien gut nachweisbar sind: „Der Teil der Wähler, der strategischen Überlegungen zugänglich ist, reagiert konsistent auf Koalitionssignale der Parteien. "23 Zur Illustration sei auf die zwei Wochen nach der Bundestagswahl 2005 durchgeführte Nachwahl im Wahlkreis 160 Dresden I verwiesen. In diesem Modellfall wurden die Wähler von den Parteien des schwarzgelben Lagers tatsächlich implizit und explizit zum Stimmensplitting aufgefordert, was im BWG ausdrücklich untersagt ist. Außerdem waren die Wähler über die Folgen ihres Handelns im Vergleich zu einer normalen Wahl außergewöhnlich gut informiert, denn die Ergebnisse der anderen 298 Wahlkreise waren bereits bekannt. Die CDU war bemüht, das als sicher betrachtete Direktmandat zu gewinnen, aber gleichzeitig die Anzahl der Zweitstimmen gering zu halten, um auf der Landesliste kein zusätzliches Mandat zu bekommen. Das sichere Direktmandat wäre in diesem Fall nicht zu einem Überhangmandat geworden, hätte die Unionsfraktion im Bundestag also nicht vergrößert. Offenbar hatte das Kalkül der CDU Erfolg: Durch geteilte Stimmabgabe stieg das Verhältnis von Erst- zu Zweitstimmen bei der CDU auf 1,52 (57931:38208), im Landesmittel lag es nur bei 1,18 (940306:795316). Spiegelbildlich dazu sank der Quotient von Erst- und Zweitstimme der FDP im Wahlkreis 160 auf 0,28 (7367:26041), während er im Landesmittel bei 0,66 (176889:269623) lag. Vom Aggregat des Wahlergebnisses im Wahlkreis Dresden I darf natürlich nicht auf individuelles Verhalten geschlossen werden, dennoch ist begründet zu vermuten, dass das Niveau des Lagersplittings aufgrund der von den Parteien deutlich 
gemachten Effekte geradezu explodiert ist - und zwar obwohl bereits absehbar war, dass Union und FDP in Berlin keine Koalition würden bilden können.

\section{Splittingszenarien: Neue Sitzverteilungen und Koalitionsoptionen}

Im Folgenden wird untersucht, wie sich die Mandatsverteilung im Bundestag veränderte, wenn Wähler der beiden Lager in größerem Ausmaß splitteten. Dafür werden Szenarien simuliert, bei denen sich innerhalb der Lager die zusätzlichen Splittinganteile von Erst- und Zweitstimmen Schritt für Schritt erhöhen, zunächst (Szenario 1 und 2) nur in jeweils einem Lager, anschließend in Szenario 3 in beiden gleichzeitig.

\subsection{Szenario 1: Sukzessive Steigerung des Stimmensplittings im schwarz-gelben Lager}

Ein verstärktes Stimmensplitting im Lager von Union und FDP führte dazu, dass für beide der Pool an Schattenmandaten so weit wie möglich ausgeschöpft würde. In der Summe nähmen die Mandate von Union und FDP durch die Erzeugung von Überhangmandaten erheblich zu. In etwa gleichem Maße vergrößerte sich auch der Bundestag, der um bis zu 176 Sitze auf 790 Sitze wüchse (siehe Tabelle 1). Die Linke, Bündnis90/Die Grünen und SPD verlören zwar in diesem Szenario keine Listenmandate. ${ }^{24}$ Durch Überhangmandate könnte Schwarz-Gelb seinen relativen Anteil an den Bundestagsmandaten aber beträchtlich steigern, im Extremfall auf über 59 Prozent - bei einem für die Proporzberechnung maßgeblichen Zweitstimmenanteil von 44,0 Prozent.

Zwar verlöre die SPD bis zu fünf Mandate, diese wären aber Überhangmandate. Sie gingen verloren, weil sie nicht durch den der SPD zustehenden Proporzanteil abgedeckt wären. Die Union könnte 38 Wahlkreise mehr direkt gewinnen. Dennoch könnte die Größe der Unionsfraktion im Bundestag im Extremfall von 226 Mandaten auf 188 Mandate sinken. Dabei kämen zwei gegenläufige Effekte zum Tragen: Zum einen verlören die Unionsparteien Listenmandate, die sich gemäß unserem Modell 1:1 im Anwachsen der FDPFraktion zeigten. Dadurch wären bei der Union immer weniger Direktmandate durch den Anteil der Zweitstimmen abgedeckt, wodurch Überhangmandate entstünden. Gleichzeitig würde die Entstehung von Überhangmandaten dadurch begünstigt, dass die Union mehr Lagererststimmen erhielte. Dadurch würde sich die Anzahl der direkt gewonnenen Wahlkreismandate auf 188 steigern. Die FDP bekäme bei einem zusätzlichen Splittinganteil von 100 Prozent alle 280 Listenmandate des schwarz-gelben Lagers. Nach dem tatsächlichen Wahlergebnis standen der Union 219 und der FDP 61 Sitze zu. Die durch die zusätzlichen Überhangmandate bedingte Vergrößerung des Bundestags ginge allein auf die Ausschöpfung des Pools an Schattenmandaten durch Schwarz-Gelb zurück. Abbildung 1 verdeutlicht noch einmal das bisher Beschriebene: SPD, Bündnis90/Die Grünen und Die Linke verlören durch verstärktes Stimmensplitting im schwarz-gelben Lager keine oder nur geringfügig Mandate. Bei der FDP würden die zusätzlichen Zweitstimmen unmittelbar und

24 Einzige Ausnahme wäre ein Sitzverlust der Grünen bei einem zusätzlichen Splittingniveau von +40 Prozent im schwarz-gelben Lager, der aus dem Umgang mit Restbruchteilen beim Verrechnungsverfahren resultierte. 


\begin{tabular}{|c|c|c|c|c|c|c|c|}
\hline $\begin{array}{l}\text { Splitting- } \\
\text { niveau } \\
\text { Schwarz- } \\
\text { Gelb }\end{array}$ & SPD & Union & FDP & $\begin{array}{c}\text { Die } \\
\text { Linke }\end{array}$ & $\begin{array}{l}\text { Bündnis } \\
90 / \\
\text { Grüne }\end{array}$ & $\begin{array}{l}\text { Bundes- } \\
\text { tagssitze }\end{array}$ & $\begin{array}{c}\text { Mehrheitsfähige } \\
\text { Zweier- } \\
\text { Koalitionen }\end{array}$ \\
\hline $\begin{array}{c}\text { Ergebnis } \\
2005\end{array}$ & 222 [9] & 226 [7] & 61 & 54 & 51 & 614 & Union/SPD \\
\hline$+10 \%$ & 222 [9] & $211[14]$ & 83 & 54 & 51 & 621 & SPD/Union \\
\hline$+20 \%$ & 221 [8] & 200 [25] & 105 & 54 & 51 & 631 & $\begin{array}{l}\text { SPD/Union } \\
\text { SPD/FDP }\end{array}$ \\
\hline$+30 \%$ & $221[8]$ & 191 [38] & 127 & 54 & 51 & 644 & $\begin{array}{c}\text { SPD/Union } \\
\text { SPD/FDP }\end{array}$ \\
\hline +40\% & 220 [7] & 184 [52] & 149 & 54 & 50 & 657 & $\begin{array}{l}\text { SPD/FDP } \\
\text { SPD/Union } \\
\text { Union/FDP } \\
\end{array}$ \\
\hline$+50 \%$ & 220 [7] & 183 [74] & 171 & 54 & 51 & 679 & $\begin{array}{l}\text { SPD/Union } \\
\text { SPD/FDP } \\
\text { Union/FDP }\end{array}$ \\
\hline$+75 \%$ & 218 [5] & $180[125]$ & 225 & 54 & 51 & 728 & $\begin{array}{l}\text { FDP/SPD } \\
\text { SPD/Union } \\
\text { FDP/Union } \\
\end{array}$ \\
\hline$+100 \%$ & 217 [4] & 188 [188] & 280 & 54 & 51 & 790 & $\begin{array}{l}\text { FDP/SPD } \\
\text { SPD/Union } \\
\text { FDP/Union } \\
\end{array}$ \\
\hline \multicolumn{8}{|c|}{$\begin{array}{l}\text { Quelle: Eigene Berechnung auf Basis des amtlichen Endergebnisses der BTW 2005. Lesehilfe für Zeile } \\
\text { „+50 \%“: Bei einem zusätzlichen Splittinganteil von } 50 \text { Prozent im schwarz-gelben Lager entfielen auf } \\
\text { die SPD } 220 \text { Mandate, davon sieben Überhangmandate. Die Union gewönne insgesamt } 183 \text { Mandate, } \\
\text { davon } 74 \text { Überhangmandate. Die FDP käme auf 171, Die Linke auf } 54 \text { und Bündnis90/Die Grünen auf } \\
51 \text { Sitze. Im dann } 679 \text { Mandate umfassenden Bundestag könnten drei mehrheitsfähige Zweier-Koalitio- } \\
\text { nen gebildet werden. Nach der Mandatszahl absteigend geordnet, sind dies Große Koalition (unter Füh- } \\
\text { rung der SPD), eine rot-gelbe und eine schwarz-gelbe Koalition. Die stärkste Koalitionsfraktion wird je- } \\
\text { weils an erster Stelle aufgeführt. }\end{array}$} \\
\hline
\end{tabular}

kontinuierlich in eine steigende Zahl von Listenmandaten umgesetzt. Die Unionsparteien könnten den Verlust an Listenmandaten durch Überhangmandate zu einem großen Teil kompensieren, so dass es zu einer drastischen Parlamentsvergrößerung käme.

Die Entwicklung der Koalitionsmöglichkeiten ist Abbildung 2 zu entnehmen. Von entscheidender Bedeutung ist die gestrichelte Mehrheitslinie: Nur Koalitionen, die oberhalb der Mehrheitslinie liegen, sind mehrheitsfähig. Durch die bei verstärktem Stimmensplitting auftretende Vergrößerung des Bundestages stiege auch die Anzahl der Mandate, die für eine Mehrheit notwendig wären. Nach dem realen Wahlergebnis liegt lediglich eine Große Koalition oberhalb der Mehrheitslinie. Da mit zunehmendem Stimmensplitting im schwarzgelben Lager die Union deutlich Mandate verliert und gleichzeitig die Mehrheitslinie steigt, würde allerdings die Mehrheit der Großen Koalition immer knapper - ganz abgesehen davon, dass sich die Stärkeverhältnisse innerhalb der Koalition dramatisch veränderten. Die rot-grüne Option verlöre absolut nur unwesentlich Parlamentssitze, entfernte sich aber immer weiter von der Mehrheitslinie, je stärker im schwarz-gelben Lager gesplittet würde diese Verluste wären vor allem relative. Mit steigendem Splittingniveau eröffneten sich neue mehrheitsfähige Koalitionsmöglichkeiten, wobei hier nur Zwei-Parteien-Koalitionen in Be- 


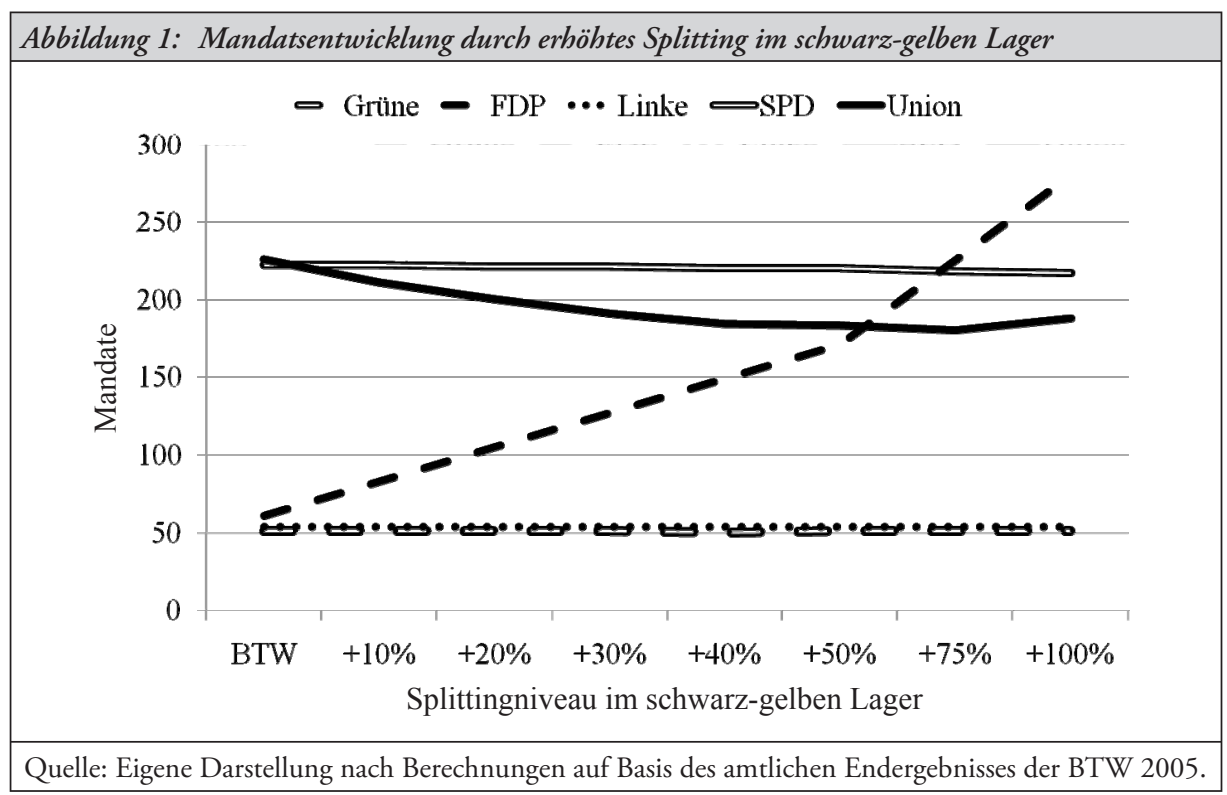

tracht gezogen und mögliche ideologische oder parteipolitische „Stolpersteine“ ausgeblendet werden.

Besonders interessant ist, dass ab einem zusätzlichen Splitting von 20 Prozent im schwarz-gelben Lager eine Koalition aus SPD und FDP möglich würde. Wir vermuten,

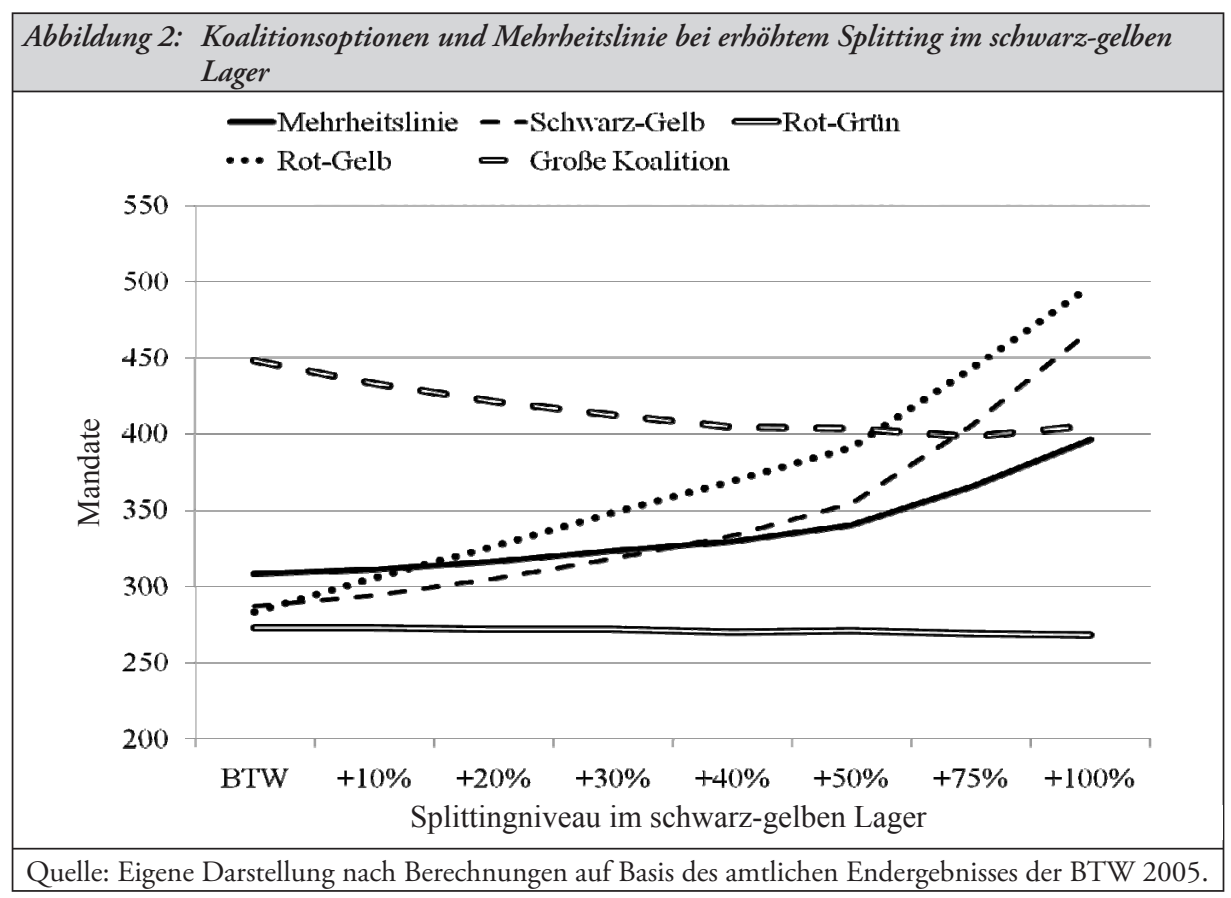


dass sich in einer solchen Situation die FDP den Werbungen der SPD nicht verschließen könnte, obwohl allein die Leihstimmen der Union diese Option ermöglicht hätten. Erst ab einem Splittingniveau von +40 Prozent wäre ein „lagerkonformes“ Bündnis aus Union und FDP mehrheitsfähig. Es würde wahrscheinlich auch zustande kommen, obwohl eine Koalition aus SPD und FDP eine deutlich komfortablere Mehrheit hätte. Schon an dieser Stelle würden Verhandlungen fast auf gleicher Augenhöhe geführt, da die Union nur noch über 184, die Freidemokraten aber bereits über 149 Mandate verfügten. Zwischen einem zusätzlichen Splittinganteil von 50 und 60 Prozent würde die FDP zum größeren Koalitionspartner, was erhebliche inhaltliche und personelle Konsequenzen für die Kabinettsbildung vermuten ließe. Spätestens ab diesem Punkt stellte sich wegen der Fraktionsstärken die Frage nach einem FDP-Kanzler. Ab einem zusätzlichen Stimmensplitting von ca. 75 Prozent würden die Freidemokraten zur stärksten Fraktion des Bundestages.

\subsection{Szenario 2: Sukzessive Steigerung des Stimmensplittings im rot-grünen Lager}

Analog zur eben gewählten Vorgehensweise soll nun ein systematisch ansteigender zusätzlicher Splittinganteil im rot-grünen Lager simuliert werden. Ausgangssituation ist wieder das tatsächliche Wahlergebnis. In diesem Szenario bleibt der Splittinganteil im Lager von Union und FDP unverändert. Es zeigt sich ein unter Lageraspekten spiegelverkehrtes, aber in Grundzügen vergleichbares Bild: Die Anzahl der Sitze bliebe bei jenen Parteien, deren Anhänger nicht stärker splitteten, fast unverändert (siehe Tabelle 2). Lediglich die Unionsparteien verlören drei Überhangmandate. Rot-Grün bediente sich schrittweise aus dem Pool von Schattenmandaten bis zu einer maximalen Anzahl von 172; in dieser Situation wären alle direkt gewonnenen SPD-Mandate Überhangmandate. Dadurch könnten Sozialdemokraten und Grüne ihren relativen Anteil an den Bundestagsmandaten im Extremfall auf 56,3 Prozent steigern - bei einem für die Proporzberechnung maßgeblichen Zweitstimmenanteil von 42,3 Prozent.

Bei der Entstehung von Überhangmandaten würden wieder die gleichen zwei modellimmanenten Mechanismen wirken: Der durch Lagersplitting bewirkte höhere Zweitstimmenanteil der Grünen verringerte direkt die Anzahl der Sitze, die den Landeslisten der SPD nach Proporz zustünden. Dadurch wären immer weniger erfolgreiche Wahlkreiskandidaten der SPD durch zustehende Listenmandate in den Ländern abgedeckt. Es entstünden Überhangmandate. Auf der anderen Seite könnte die SPD durch zusätzliche Lagererststimmen 27 Wahlkreise mehr gewinnen, die zu Überhangmandaten würden, sobald sie nicht mehr durch die Zweitstimmenanteile der Landeslisten abgedeckt wären. Wie schon beim vorigen Szenario veränderte sich die Anzahl der durch Proporz zugeteilten Bundestagsmandate für das rot-grüne Lager nicht. Die 213 „regulären“ Mandate der SPD gesellten sich durch den Zweitstimmentransfer sukzessive zu den tatsächlichen 51 Mandaten von Bündnis90/Die Grünen bis zu einem Maximum von 264 Sitzen. Bündnis90/Die Grünen würden bei einem etwa 80 Prozent erhöhten Splittingniveau, wie schon die Freidemokraten im vorherigen Szenario, zur stärksten Fraktion im Bundestag aufsteigen. Nach Proporz würden keine zusätzlichen Mandate verteilt. Dennoch würde sich der Bundestag auf 774 Mandate vergrößern, weil dem rot-grünen Lager aus dem Pool der Schattenmandate Sitze zugeteilt würden. 


\begin{tabular}{|c|c|c|c|c|c|c|c|}
\hline \multicolumn{7}{|c|}{ Tabelle 2: Folgen von erhöhtem Splitting im rot-grünen Lager } \\
\hline $\begin{array}{c}\text { Splitting- } \\
\text { niveau } \\
\text { Rot-Grün }\end{array}$ & SPD & Union & FDP & $\begin{array}{c}\text { Die } \\
\text { Linke }\end{array}$ & $\begin{array}{c}\text { Bünd- } \\
\text { nis90/ } \\
\text { Grüne }\end{array}$ & $\begin{array}{c}\text { Bundes- } \\
\text { tagssitze }\end{array}$ & $\begin{array}{c}\text { Mehrheitsfähige } \\
\text { Zweier- } \\
\text { Koalitionen }\end{array}$ \\
\hline $\begin{array}{c}\text { Ergebnis } \\
\mathbf{2 0 0 5}\end{array}$ & $222[9]$ & $226[7]$ & 61 & 54 & 51 & 614 & Union/SPD \\
\hline$+\mathbf{1 0} \%$ & $205[13]$ & $224[5]$ & 61 & 54 & 72 & 616 & Union/SPD \\
\hline$+\mathbf{2 0} \%$ & $194[23]$ & $224[5]$ & 61 & 54 & 93 & 626 & $\begin{array}{c}\text { Union/SPD } \\
\text { Union/Grüne }\end{array}$ \\
\hline$+\mathbf{3 0} \%$ & $186[37]$ & $223[4]$ & 61 & 54 & 115 & 639 & $\begin{array}{c}\text { Union/SPD } \\
\text { Union/Grüne }\end{array}$ \\
\hline$+\mathbf{4 0} \%$ & $180[52]$ & $223[4]$ & 61 & 54 & 136 & 654 & $\begin{array}{c}\text { Union/SPD } \\
\text { Union/Grüne }\end{array}$ \\
\hline$+50 \%$ & $176[69]$ & $223[4]$ & 61 & 54 & 157 & 671 & $\begin{array}{c}\text { Union/SPD } \\
\text { Union/Grüne }\end{array}$ \\
\hline$+75 \%$ & $170[117]$ & $223[4]$ & 61 & 54 & 211 & 719 & $\begin{array}{c}\text { Union/Grüne } \\
\text { Union/SPD } \\
\text { Grüne/SPD }\end{array}$ \\
\hline$+100 \%$ & $172[172]$ & $223[4]$ & 61 & 54 & 264 & 774 & $\begin{array}{c}\text { Union/Grüne } \\
\text { Grüne/SPD } \\
\text { Union/SPD }\end{array}$ \\
\hline
\end{tabular}

Im Vergleich der beiden Szenarien zeigt sich, dass das Potential zur Aufblähung des Bundestages durch das bürgerliche Lager größer ist als durch das rot-grüne, und zwar genau in dem Maße, in dem die Union mehr Wahlkreise gewinnen könnte als die SPD. Dies verdeutlicht, dass erfolgreiche Wahlkreiskandidaten der Schlüssel für Mandatsmehrheiten ohne Stimmenmehrheiten sind. Gleichwohl profitierte in beiden Szenarien bei einem sehr hohen zusätzlichen Splittinganteil die ursprünglich kleine Lagerpartei. Die koalitionspolitischen Optionen und Gewichte verschöben sich dramatisch.

Die Betrachtung der Koalitionsmöglichkeiten bei verstärktem rot-grünen Lagersplitting liefert vergleichbare Ergebnisse wie schon zuvor das verstärkte Splitting bei Schwarz-Gelb. Auch hier wäre zu Anfang nur eine Große Koalition möglich, die aber deutlich an absoluter und relativer Größe verlöre. Absolute Mandatszahl einer möglichen Großen Koalition und Mehrheitslinie nähern sich immer weiter an, bis eine Große Koalition bei einem Splittingniveau von +100 Prozent nur noch eine knappe Mandatsmehrheit im Parlament hätte. Bemerkenswert ist, dass sich die Stärkeverhältnisse innerhalb der Option Große Koalition deutlich zugunsten der Union veränderten, weil die SPD durch höhere Splittingniveaus im rot-grünen Lager Mandate verlöre. Union und FDP behielten, ähnlich dem rot-grünen Lager im vorigen Szenario, zwar absolut annähernd gleich viel Mandate, entfernten sich aber immer weiter von der Mehrheitslinie und büßten damit auch relativ an Einfluss im Bundestag ein - etwa bei der Besetzung von Ausschüssen. Erst ab einem erhöhten Splittingniveau von über 20 Prozent ergäben sich alternative Mehrheitsverhältnisse neben der Großen Koalition. Analog zum vorigen Szenario wäre auch hier die erste alternative Möglichkeit einer Zwei-Parteien-Koalition jenseits der etablierten Lager: Ab circa 20 Prozent zusätzlichem Splitting wäre Schwarz-Grün mehrheitsfähig. Bis gut +50 Prozent Splittingniveau bliebe diese Option die einzige Alternative zur Großen Koalition. Ab einem zusätzli- 
chen Stimmensplitting von knapp über 50 Prozent im rot-grünen Lager könnte aus der rot-grünen Vorwahlkoalition ein reales mehrheitsfähiges Bündnis gebildet werden. Schon ab etwa +60 Prozent wären die Grünen nicht mehr nur ein gleichberechtigter Partner, sondern würden auch die größere Fraktion innerhalb der Koalition stellen - mit erwartbaren Effekten auf die Besetzung des Kanzleramtes.

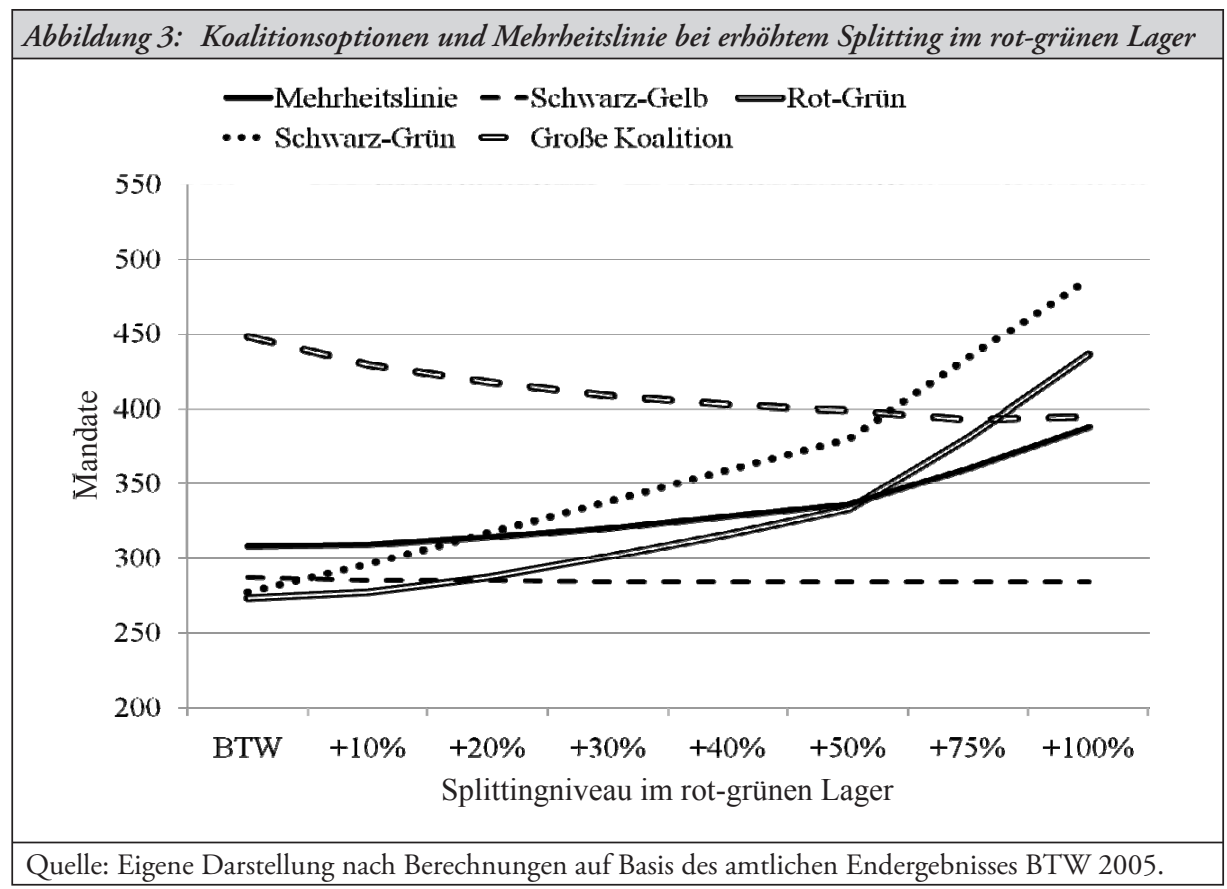

\subsection{Szenario 3: Parallele Steigerung des Stimmensplittings in beiden Lagern}

Die systematische Anhebung der Splittinganteile in beiden Lagern scheint ein plausibleres Szenario als die eben diskutierten zu sein, würde den Erfahrungen der letzten 25 Jahre entsprechen und die bisherige Entwicklung fortschreiben. Auffällig ist, dass die Mandatszahlen von FDP und Bündnis90/Die Grünen genau in dem Maße zunehmen würden, wie sie auch schon in den jeweiligen Szenarien mit verstärktem Splitting in nur einem Lager gewachsen wären (siehe Tabelle 3). Die Mandatszahlen für SPD und Union sänken kontinuierlich, da die Verluste von Listenmandaten durch abnehmende Zweitstimmenanteile nicht vollständig durch Direktmandate kompensiert werden könnten: Im Vergleich zu den beiden anderen Szenarien gewönnen bei einem Splittingniveau von +100 Prozent SPD und Union in der Summe bis auf eine Ausnahme keine zusätzlichen Wahlkreise. Auch der relative Anteil der beiden Lager an den Bundestagsmandaten würde gegenüber ihrem Zweitstimmenanteil nur noch geringfügig ansteigen: auf etwa 48 Prozent für Schwarz-Gelb und circa 46 Prozent für Rot-Grün. Kein Lager könnte in diesem Szenario eine mehrheitsfähige Koalition bilden. 


\begin{tabular}{|c|c|c|c|c|c|c|c|}
\hline \multicolumn{7}{|c|}{ Tabelle 3: Folgen von erhöhtem Splitting in beiden Lagern } \\
\hline $\begin{array}{c}\text { Splitting- } \\
\text { niveau in } \\
\text { beiden } \\
\text { Lagern }\end{array}$ & SPD & Union & FDP & $\begin{array}{c}\text { Die } \\
\text { Linke }\end{array}$ & $\begin{array}{c}\text { Bünd- } \\
\text { nis90/ } \\
\text { Grüne }\end{array}$ & $\begin{array}{c}\text { Bundes- } \\
\text { tagssitze }\end{array}$ & $\begin{array}{c}\text { Mehrheitsfähige } \\
\text { Zweier- } \\
\text { Koalitionen }\end{array}$ \\
\hline $\begin{array}{c}\text { Ergebnis } \\
\mathbf{2 0 0 5}\end{array}$ & $222[9]$ & $226[7]$ & 61 & 54 & 51 & 614 & Union/SPD \\
\hline$+\mathbf{1 0} \%$ & $205[13]$ & $209[12]$ & 83 & 54 & 72 & 623 & Union/SPD \\
\hline$+\mathbf{2 0} \%$ & $190[19]$ & $197[22]$ & 105 & 54 & 93 & 639 & Union/SPD \\
\hline$+\mathbf{3 0} \%$ & $183[34]$ & $185[32]$ & 127 & 54 & 115 & 664 & Union/SPD \\
\hline$+\mathbf{4 0} \%$ & $175[47]$ & $174[43]$ & 149 & 54 & 136 & 688 & SPD/Union \\
\hline$+\mathbf{5 0} \%$ & $169[62]$ & $165[56]$ & 171 & 54 & 157 & 716 & $\mathrm{keine}$ \\
\hline$+\mathbf{7 5} \%$ & $151[98]$ & $153[98]$ & 225 & 54 & 211 & 794 & FDP/Grüne \\
\hline$+\mathbf{1 0 0} \%$ & $146[146]$ & $150[150]$ & 280 & 54 & 264 & 894 & FDP/Grüne \\
\hline Quelle: Eigene Berechnung auf Basis des amtlichen Endergebnisses der BTW 2005. & \\
\hline
\end{tabular}

Im Schatten der Stimmen- und Mandatsverrechnung entstünden allerdings mandatsrelevante Bewegungen, denn die Wahlkreissieger wären in einigen Fällen andere als die im Wahlergebnis 2005 ausgewiesenen. In Baden-Württemberg verlöre zum Beispiel die CDU im Saldo vier Wahlkreise. Im Vergleich zu den beiden anderen Szenarien, in denen SPD und Union auf Kosten des jeweils anderen massiv zusätzlich Direktmandate gewönnen, käme es bei parallelem Ansteigen der Lagersplittingniveaus zu einer Nivellierung. Anzumerken bleibt noch, dass Die Linke alle drei Direktmandate in allen Szenarien behielte, was keine Selbstverständlichkeit ist. Vielmehr wäre zu erwarten, dass Parteien, die keinem Lager angehören, aufgrund der gestiegenen relativen Mehrheitsschwelle, die durch die Konzentration der Lager-Erststimmen bewirkt würde, Direktmandate verlören. Bei einem um 100 Prozent erhöhten Splittingniveau würden die 598 Mandate, die nach dem Verhältnis der Zweitstimmen vergeben werden, nur noch auf drei Parteien verteilt, nämlich auf FDP, Bündnis90/Die Grünen und Die Linke. Dabei vereinten FDP und Bündnis90/Die Grünen alle auf ihr Lager entfallenden Listenmandate. Auch hier erhöhte sich die Zahl der zugewiesenen Proporzmandate in der Summe für die jeweiligen Lager nicht. Die Aufblähung des Parlaments erfolgte allein durch die Umwandlung aller von den beiden Lagern gewonnenen Direktmandate in Überhangmandate. Der Pool von 299 möglichen Schattenmandaten würde fast vollständig (bis auf drei Mandate) ausgeschöpft.

Mit Blick auf die Koalitionsmöglichkeiten fällt auf, dass - anders als in den beiden oben diskutierten Szenarien - sich mehrheitsfähige Zweierkoalitionen jenseits der Großen Koalition erst bei einem sehr hohen zusätzlichen Splittingniveau ergeben. Eine Große Koalition wäre außerdem nur bis zu einem Splittingniveau von circa +40 Prozent mehrheitsfähig, wobei in diesem Fall die SPD zur größeren Koalitionspartei würde. Bei einem Splittinganteil von +50 Prozent wäre kein Zweierbündnis mehrheitsfähig. Die einzige sich bei noch höheren Splittingniveaus (über +75 Prozent) ergebende Zweierkoalition könnte von FDP und Bündnis90/Die Grünen gebildet werden (siehe Abbildung 4).

Insgesamt würden sich im Extremfall völlig andere Mehrheitsverhältnisse im Bundestag ergeben. Die Rangfolge der Fraktionsstärken würde fast vollständig auf den Kopf gestellt. Die beiden großen Volksparteien würden nur die dritt- (Union) und viertstärkste (SPD) 
Fraktion bilden und wären für eine Regierungsbildung entbehrlich. Dagegen wären FDP und Bündnis90/Die Grünen die „großen“ parlamentarischen Akteure. Wesentliche Effekte zeigen sich aber auch jenseits der beiden Lager. Die Linke behielte absolut alle Mandate, verlöre aber relativ: Die relative Fraktionsstärke würde von 8,8 Prozent (BTW 2005) auf sechs Prozent absinken. Der relative Einflussverlust entspräche ungefähr einem Drittel und damit etwa dem Anteil der Überhangmandate im Parlament. Dies macht deutlich, dass Parteien, die keinem Lager angehören, durch Lagersplitting an Einfluss verlieren könnten. Außerdem wäre bei einer starken Parlamentsaufblähung der Fraktionsstatus kleiner Parteien in Gefahr - mit Auswirkungen auf Mittelzuweisungen und parlamentarische Rechte.

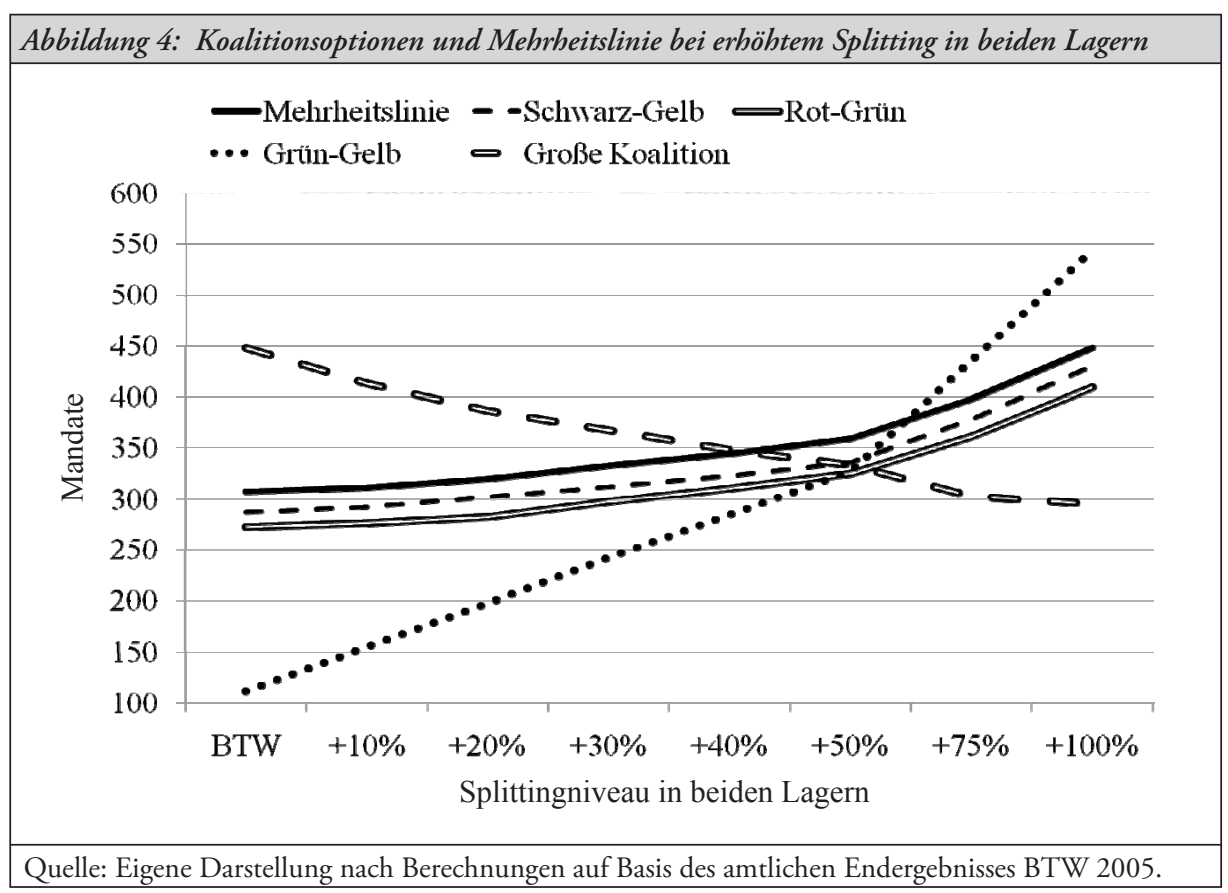

\section{Wahl-und verfassungsrechtliche Implikationen}

Aus den vorgestellten Szenarien über verstärktes Stimmensplitting in Parteilagern ist klar ersichtlich, dass eine Zunahme geteilter Stimmabgabe erhebliche Effekte auf die Sitzverteilung im Bundestag und damit auf Regierungsbildung und politische Entscheidungen haben kann. Ursache für die variable Sitzverteilung bei gleicher Unterstützung der Lager durch das Elektorat ist die Ausschöpfung des Pools an Schattenmandaten, die als Überhangmandate den Bundestag aufblähen. Das im Wahlsystem auf Bundesebene bestehende Einfallstor der Überhangmandate wird durch stärkeres Lagersplitting weit aufgestoßen. Die Mandatsanteile und das Stärkeverhältnis der Fraktionen können durch steigende Splittingniveaus nicht nur verändert, sondern in Extremfällen sogar auf den Kopf gestellt werden. Kippende Mehrheiten und alternative Koalitionsmöglichkeiten sind in den hier vorgestellten Szenarien keine Ausnahme, sondern die Regel. 
Die hohe Varianz in der Mandatszahl eines Lagers bei faktisch gleicher Wählerunterstützung für die Lager ist nach unserer Auffassung in einem Verhältniswahlsystem nicht hinnehmbar. Die Schwankungsbreite ist enorm: Bei einem Zweitstimmenanteil von 42,3 Prozent entfielen auf Rot-Grün in Szenario 1 bei einem um 100 Prozent erhöhten Splittingniveau des schwarz-gelben Lagers lediglich 33,9 Prozent der Bundestagsmandate. Dagegen würde der Mandatsanteil in Szenario 2 auf bis zu 56,3 Prozent anwachsen können, wenn im rot-grünen Lager total, im schwarz-gelben dagegen gar nicht zusätzlich gesplittet würde. Analog schwankt auch der Mandatsanteil von Schwarz-Gelb zwischen 59,0 Prozent in Szenario 1 und 36,7 Prozent in Szenario 2.

Das Bundesverfassungsgericht hat in seiner Entscheidung zum negativen Stimmgewicht unmissverständlich darauf hingewiesen, dass der in Art. 38 Abs. 1 GG verankerte Gleichheitsgrundsatz „im Sinne einer strengen und formalen Gleichheit zu verstehen“ sei, also jede abgegebene Stimme denselben Zählwert und dieselbe rechtliche Erfolgschance haben muss. Die Lösung des durch massive Ausnutzung des Schattenmandatspools entstehenden Problems kann also nur in der Verhinderung, Abschaffung oder Kompensation von Überhangmandaten liegen. In diesem Zusammenhang ergeben sich drei mögliche Modifizierungen des Wahlsystems, die hier kurz skizziert werden sollen. ${ }^{25}$

Verrechnung der Direktmandate auf der Bundesebene: Entweder werden alle gewonnenen Direktmandate von der Anzahl der Mandate abgezogen, die einer Partei zustehen, oder es werden Ausgleichsmandate eingeführt. Nach der ersten Variante sollten die Parteien mit Bundeslisten zur Wahl antreten. Bei der Verteilung der Mandate auf die einzelnen Bundesländer ergäben sich dann aber Verzerrungen. Dies könnte dazu führen, dass einzelne Landesverbände bestimmter Parteien gegenüber anderen Landesverbänden bevorzugt behandelt würden, nämlich jene, in denen traditionell viele Direktmandate gewonnen werden oder Überhangmandate entstehen. Die Wahl von Parteibundeslisten wäre aber angesichts der föderalen Gliederung der Parteien in Deutschland schwer zu realisieren. Bei der Einführung von Ausgleichsmandaten bei Bundestagswahlen stellte sich dagegen die Frage nach der Anzahl beziehungsweise der Anzahl der Iterationsschritte und ob der Ausgleich auf Bundesebene oder auf Bundes- und Landesebene erfolgen soll. Ohne genauer auf das Verfahren einzugehen, steht fest, dass Ausgleichsmandate zwei wesentliche Nachteile haben: Zum einen blähten sie das Parlament auf, das bereits um die Überhangmandate vergrößert wäre. ${ }^{26}$ Zum anderen wird der Stimmenverrechnungsmechanismus noch komplizierter und für den Wähler undurchsichtiger. Darüber hinaus wäre bei einer geringen Anzahl von Ausgleichs-

25 Ähnliche Vorschläge skizziert Joachim Behnke, a.a.O. (Fn. 5), S. 515. Auf andere Wahlrechtsreformvorschläge, die das Mehrheitselement stärken, wird hier nicht eingegangen, da die Intention genau entgegengesetzt zur Abschaffung der Überhangmandate ist, die das Verhältniselement stärken. Den Vorschlag, Überhangmandate durch eine Neuinterpretation des BWG in der Praxis abzuschaffen, findet man bei Christian Naundorf, Der überflüssige Überhang: Reformvorschläge, in: ZParl, 27. Jg. (1996), H. 3, S. 393 - 397.

26 Um zum Beispiel die vier der SPD in Sachsen-Anhalt bei der Bundestagswahl 2005 entstandenen Überhangmandate zu kompensieren, hätten so lange Ausgleichsmandate geschaffen werden müssen, bis auf die SPD-Landesliste zehn Mandate entfallen wären. Da der SPD-Landesliste nur sechs Mandate zustanden, hätte der Bundestag um Zweidrittel vergrößert werden müssen. In den Extremfällen der von uns diskutierten Szenarien würden Iterationsverfahren nicht mehr konvergieren. Weil bei SPD und Union keine Zweitstimmenanteile verrechnet werden könnten, würde der Kompensationsprozess uferlos. 
mandaten beziehungsweise Iterationsschritten nicht gesichert, dass Effekte wie der des negativen Stimmgewichts neutralisiert würden.

Senkung des Verhältnisses von Wablkreis- und Listenmandaten: Aktuell werden nach dem Verrechnungsverfahren die 299 Direktmandate mit den 598 Mandaten, die den Parteien nach Proporz zugeteilt werden, verrechnet. Ohne Überhangmandate sollte die Hälfte der nach Proporz zugeteilten Mandate durch erfolgreiche Wahlkreiskandidaten besetzt werden. Eine relativ elegante Möglichkeit, das Entstehen von Überhangmandaten zu erschweren, besteht darin, dieses Verhältnis abzusenken. ${ }^{27}$ Bei einem Anteil von einem Drittel würde beispielsweise in Sachsen-Anhalt nur noch ein Überhangmandat für die SPD entstehen, anstatt der vorherigen vier. Bei einem Anteil von einem Viertel hätte es bei dem Wahlergebnis von 2005 keine Überhangmandate mehr gegeben. ${ }^{28}$ Die Senkung des Verhältnisses von Wahlkreis- und Listenmandaten hat einen entscheidenden Vorteil gegenüber den anderen Modifikationen des Wahlsystems: Das bisherige Verrechnungsverfahren könnte beibehalten werden. Wenn alle Überhangmandate wegfielen, würde sich auch der Effekt des negativen Stimmgewichts verflüchtigen. Zu klären wäre lediglich, ob der Bundestag vergrößert oder die Anzahl der Wahlkreise reduziert werden sollte. Dafür müssten Wahlkreise neu zugeschnitten werden, die mehr Wahlberechtigte umfassen. ${ }^{29}$ Da diese Modifikation des Wahlsystems den technischen Status quo am wenigsten änderte, aber dennoch die Entstehung von Überhangmandaten bremsen würde, erscheint uns die Umsetzungswahrscheinlichkeit dieses Ansatzes am größten. Vollständig verhindert würden Überhangmandate jedoch nicht - bei starken Splittingniveaus wie in unseren Szenarien könnten Überhangmandate weiterhin entstehen. Weil der Pool an Schattenmandaten aber erheblich kleiner ausfiele, würden die proporzverzerrenden Effekte von Lagersplitting abgeschwächt. Gleichzeitig ließen sich geringere Anreize zum Splitting vermuten. ${ }^{30}$

System einer lose gebundenen Liste: Überhangmandate (und damit ungleiches oder negatives Stimmgewicht) sind das Resultat der Verrechnung von Erst- und Zweitstimmen. Sie könnten am einfachsten (und vollständig) durch die Etablierung eines Verhältniswahlsys-

27 An dieser Stellschraube lässt sich auch in die andere Richtung drehen, wie Gerd Strohmeier vorschlägt. Die Begünstigung der Entstehung von Überhangmandaten hat dann einen mehrheitsbildenden Effekt, ders., Ein Plädoyer für die „gemäßigte Mehrheitswahl“: optimale Lösung für Deutschland. Vorbild für Österreich und andere Demokratien, in: ZParl, 38. Jg. (2007), H. 3, S. $578-590$.

28 Würde der Anteil der durch Proporz vergebenen Mandate auf ein Drittel abgesenkt, stünden der Landesliste neun Mandate zu (bei konstant gehaltener Zahl der Wahlkreise). Die SPD bekäme in Sachsen-Anhalt nur noch ein Überhangmandat. Bei einer Absenkung auf einen Anteil von einem Viertel würden der SPD-Landesliste zwölf Mandate zustehen, wodurch kein Überhangmandat mehr entstünde.

29 Der Neuzuschnitt von Wahlkreisen ist keinesfalls trivial. Technisch müssen die Grenzen regionaler Gliederungen bei möglichst einheitlicher Bevölkerungszahl berücksichtigt werden. Bei zu großen Wahlkreisen nimmt die Rückbindung der Abgeordneten an ihre Kreise ab, womit der Sinn der Personalisierung verwässert würde.

30 In eine ähnliche Richtung zielt die von Joachim Behnke vorgeschlagene Einführung von Doppelwahlkreisen. Sie verringern die Wahrscheinlichkeit, dass eine Partei in einem Bundesland (fast) alle der möglichen Direktmandate gewinnt, wenn diese nur geringfügig stärker ist als die Konkurrenz. Vorteilhaft bei dieser Variante wäre, dass (ohne Überhangmandate) nach wie vor die Hälfte der Mandate durch erfolgreiche Wahlkreiskandidaten besetzt würde. Ders., Das Wahlsystem der Bundesrepublik Deutschland: Logik, Technik und Praxis der Verhältniswahl, Baden-Baden 2007, S. 225 ff. 
tems mit nur einer Stimme abgeschafft werden. Zudem könnte auf ein beliebiges der diskutierten Proporzberechnungsverfahren zurückgegriffen werden. Eine wesentliche Veränderung gegenüber dem aktuellen Wahlsystem wäre die Entpersonalisierung der Wahl: Die Parteien, die zur Wahl antreten, entschieden alleine darüber, wen sie in das Parlament entsenden, wodurch der Wähler keinen Einfluss auf die personelle Zusammensetzung des Bundestages hätte. Dem könnte durch die Wahl einer lose gebundenen Liste entgegengewirkt werden, bei der die Rangfolge der Kandidaten auf der gewählten Landesliste beeinflusst werden kann. Der wesentliche Vorteil dieses Verfahrens gegenüber der personalisierten Verhältniswahl besteht in der besseren Verständlichkeit für den Wähler, der die Wirkung seiner Stimme personalisieren und wesentlich einfacher nachvollziehen kann. Weil eine lose gebundene Liste sehr einfach und transparent ist, den im Grundgesetz festgeschriebenen Anforderungen - im Gegensatz zum aktuellen Wahlrecht - vollständig gerecht wird und weder Überhangmandate noch Paradoxien zulässt, sollte dieses Verfahren bei der anstehenden Wahlrechtsreform implementiert werden.

\section{Wahlsiege ohne Stimmenmehrheit}

Die Wähler, in erster Linie auch viele Lagersplitter, dürften ebenso wie die Parteien von den proporzverzerrenden Splittingeffekten, insbesondere von der Möglichkeit, dass Wahlsiege ohne Mehrheiten zustande kommen, überrascht werden. Zwar können beide Lager erheblich von einer Erhöhung des Splittingniveaus profitieren; die Mandatsgewinne verteilen sich allerdings asymmetrisch: Während den großen Volksparteien bei stärkerem Splitting Mandatsverluste entstünden, würden die ursprünglich „kleinen“ Lagerparteien FDP und Bündnis90/Die Grünen in Extremfällen Fraktionen bilden können, die fast doppelt so groß wie die von Union und SPD sind. Die „Vorteile“ höherer Splittingniveaus kämen also in erster Linie Freidemokraten und Grünen zugute. Am bedeutsamsten erscheint der Umstand, dass sich eine Reihe von neuen Möglichkeiten der Regierungsbildung ergäbe, wobei hier nur mehrheitsfähige Koalitionen mit zwei Partnern berücksichtigt wurden. Eine Koalition aus SPD und Unionsparteien würde in jedem Szenario bei einer Erhöhung des Splittingniveaus Mandatsverluste erleiden, außerdem käme es mehrfach zu Führungswechseln. In Szenario 3 würde die Große Koalition am Ende sogar über weniger als die Hälfte der Bundestagsmandate verfügen. Insgesamt können sich in den Szenarien, je nach Splittingniveau, elf unterschiedliche Zweierkoalitionen mit vier unterschiedlichen Kanzlern ergeben. Ob der Kanzler von der Union (in drei Fällen), der SPD (in drei Fällen), der FDP (in drei Fällen) oder von Bündnis90/Die Grünen (in zwei Fällen) gestellt würde, wäre allein vom Niveau des Lagersplitting abhängig.

Strategisch rationales Stimmensplitting in politischen Lagern kann die Zahl der Bundestagssitze drastisch erhöhen, Mehrheiten verändern und die Koalitionsarithmetik durcheinanderwirbeln. Selbst wenn die Persistenz der Lager angesichts aktueller Entwicklungen im Parteiensystem bezweifelt werden kann, ist zu vermuten, dass bei einer Offenlegung der mandatsrelevanten Folgen von verstärktem Stimmensplitting die Wähler noch häufiger ihre Erst- und Zweitstimmen gezielt auf politisch nahestehende Parteien verteilen dürften. Sicher ist, dass das deutsche Wahlsystem in seiner bestehenden Form zahlreiche Überraschungen bereit hält, von denen hier nur einige simuliert wurden. Die Fraktionsstärken im Bundestag sind in unseren Berechnungen in erster Linie davon abhängig, in welchem Umfang 
die großen Lagerparteien den wahlsystematisch angelegten Pool an Schattenmandaten ausschöpfen können. Auch wenn stark erhöhte Splittingniveaus wenig realistisch erscheinen, sind die potentiellen Folgen im deutschen Wahlrecht bereits angelegt. Bei einer Bundestagswahl geht es eben immer auch um (derzeit 299) Schattenmandate. Bei anstehenden Reformüberlegungen sollte deshalb die Verhinderung von Überhangmandaten zum Ausgangspunkt gemacht werden - dies schüfe mehr Transparenz, stellte wahlsystematische Konsistenz her und die Erfolgswertgleichheit der Wählerstimmen sicher.

\title{
„Die Ewigkeit dauert lange, besonders gegen Ende“ - eine rechtliche (Neu-)Bewertung des kommunalen Wahlrechts für Drittstaatsangehörige
}

\author{
Felix Hanschmann
}

Nachdem das Bundesverfassungsgericht (BVerfG) 1989 die Einführung des kommunalen Ausländerwahlrechts in Schleswig-Holstein und Hamburg für verfassungswidrig erklärt hatte $^{1}$, verebbte auch die zuvor extensiv geführte Diskussion um demokratische Partizipationsrechte für Nichtdeutsche. ${ }^{2}$ Die 1992 im Zuge der Ratifikation des Vertrages über die Europäische Union erfolgte Einführung des aktiven und passiven Wahlrechts für Unionsbürger, das durch die Kommunalwahlrichtlinie 94/80/EG des Rates vom 19. Dezember $1994^{3}$ konkretisiert und für Deutschland durch Änderung des Art. 28 Abs. 1 S. 3 GG verfassungsrechtlich umgesetzt wurde ${ }^{4}$, ist von der deutschen verfassungsrechtlichen Literatur und den Medien zumindest im Hinblick auf die Streitpunkte der vorangegangenen Diskussion sowie die Prämissen der bundesverfassungsgerichtlichen Rechtsprechung kaum erörtert

1 BVerfGE 83, S. 37 ff., S. $60 \mathrm{ff}$.

2 Einen guten Überblick über die Debatte gibt die Dokumentation der beiden Verfahren, in: Josef Isensee / Edzard Schmidt-Jortzig (Hrsg.), Das Ausländerwahlrecht vor dem Bundesverfassungsgericht, Heidelberg 1993. Umfassend und mit zahlreichen weiteren Nachweisen auch Astrid Wallrabenstein, Das Verfassungsrecht der Staatsangehörigkeit, Baden-Baden 1999, S. 90 ff.

3 ABl. 1994 L 368 vom 31. Dezember 1994, S. 38 - 47. Umfassend zu dieser Richtlinie Nikolaos Kotalakidis, Von der nationalen Staatsangehörigkeit zur Unionsbürgerschaft, Baden-Baden 2000, S. 179 ff.; Peter Oliver, Electoral Rights under Article 8B of the Treaty of Rome, in: CMLRev. 1996, S. 473 - 498, S. 489 ff.; Ludger Schrapper, Die Richtlinie 94/80/EG zum aktiven und passiven Kommunalwahlrecht für Unionsbürger, in: DVBl. 1995, S. 1167 ff. Mittlerweile ist die Kommunalwahlrichtlinie von allen Bundesländern umgesetzt worden. Hierzu Meinhard Hilf, in: Eberhard Grabitz / ders. (Hrsg.), EU-Kommentar (17 EL Januar 2001), Art. 19 Rn. 22; Bodo Pieroth / Markus Schmülling, Die Umsetzung der Richtlinie des Rates zum Kommunalwahlrecht der Unionsbürger in den deutschen Ländern, in: DVBl. 1998, S. 365 ff.; Regine Schunda, Das Wahlrecht von Unionsbürgern bei Kommunalwahlen in Deutschland, Frankfurt am Main u.a. 2003, S. 282 ff. Speziell zur Umsetzung in den Stadtstaaten Klaus Sieveking, Europäisches Gemeinschaftsrecht und Stadtstaaten - verfassungsrechtliche Probleme bei der Einführung des kommunalen Ausländerwahlrechts, in: DÖV 1993, S. 449 ff.; Regine Schunda, a.a.O. (Fn. 3), S. 341 ff.

4 Eingefügt durch das 38. Gesetz zur Änderung des Grundgesetzes vom 21. Dezember 1992, BGBl. I, S. 2086. 